\title{
Long-term durability antibacterial microcapsules with plant-derived Chinese nutgall and their applications in wound dressing
}

https://doi.org/10.1515/epoly-2019-0027

Received October 14, 2018; accepted December 14, 2018.

\begin{abstract}
Wound infection is a significant burden on public health. Most present antibacterial agents are typically toxic and devoid of long-term durability. We reported an antimicrobial microcapsule with Chinese nutgall (CN) encapsulated, which was a plant-derived extraction. It is biocompatible and has been used in traditional medicine systems. Sodium alginate (SA) and chitosan worked as shells. The promise of the design is to adopt biocompatible natural polymers and electrostatic attractive chitosan and SA form stable shells to keep long-term release of $\mathrm{CN}$. The results exhibited microcapsules with integrated performance of biocompatibility, long-term durability (inhibition rate of $98.99 \%$ against $S$. aureus after $12 \mathrm{~h}$ and $100 \%$ after $12 \mathrm{~h}, 99.61 \%$ against $E$. coli after $6 \mathrm{~h}$ and $100 \%$ after $12 \mathrm{~h}$ ), high antibacterial efficacy (with $S$. aureus inhibition zones of $7.67 \mathrm{~mm}$ and $E$. coli inhibition zones of $5.27 \mathrm{~mm})$ and ease of storage $\left(-20^{\circ} \mathrm{C}\right.$ for more than $\left.60 \mathrm{~h}\right)$. Their successful fabrication may provide new insights into application of traditional cotton gauze in a sustainable and multifunctional form.
\end{abstract}

Keywords: microcapsules; antibacterial; durability; Chinese nutgall

\footnotetext{
* Corresponding author: Fujun Wang, Key laboratory of Textile Science and Technology, Ministry of Education, China; College of Textiles, Donghua University, Shanghai 201620, China, e-mail: wangfujun@dhu.edu.cn. Tel. +862167792326 Wen Xue, Mengxing Zhang, Fan Zhao, Jing Gao and Lu Wang, Key laboratory of Textile Science and Technology, Ministry of Education, China; College of Textiles, Donghua University, Shanghai 201620, China, e-mail: xuewen9256@163.com (W. X.), 1508172976@qq.com (M. Zh.), zhaofan_dhu@163.com (F. Zh.), gao2001jing@dhu.edu.cn (J.G.), wanglu@dhu.edu.cn (L. W.).
}

\section{Introduction}

Bacterial infection of open wounds constitutes the forefront of injury treatments. Wound beds provide a moist, warm and nutritious environment for microbial growth. Among four time-dependent wound healing phases, which are hemostasis, inflammation, proliferation and remodeling, the inflammation phase is the riskiest for bacteria attack (1). Various substances like toxins and proteases can cause prolonged inflammatory response of the host tissue, interfering with the wound healing process (2). To prevent the microbial infection, various antibacterial agents have been applied on the wound, including quaternary ammonium salts and silver nanoparticles (3). Although these agents can significantly minimize wound infections, they cannot satisfy wound healing needs as they still exhibit some toxicity to human beings. Besides, their immediate biocidal effects may suffer from significant decrease due to the irreversible consumptions, which work by direct contact killing (4). Thus, nontoxic and prolonged antimicrobial systems to provide protections from bacterial infections are needed.

A desirable design of antimicrobial systems requires an optimization of two important properties: the biocompatibility of antibacterial agents and the durability of biocidal functions, which affects their long-term usage. There is a growing interest in adopting natural antibacterial compounds such as extracts from herbs and plants for defending microbe because of their biocompatibility. Besides, the relatively low frequency of infectious diseases in plants suggests these natural defense mechanisms can be very effective (5). Cisowska A. presented the antimicrobial properties of anthocyanin and its identified compounds. Generally, anthocyanins are active against different microbes, and Gram-positive bacteria usually are more susceptible to the anthocyanin action than Gram-negative ones (6). H.J.D. Dorman studied the antibacterial activity of plant volatile oils from 
black pepper, geranium, nutmeg and oregano. The results demonstrated the plant volatile oils had great potentials as biocidal agents (7). However, the antibacterial function did not last long because of volatilization. Drug delivery technology by incorporating biocidal agents into hydrogels could be a strategy and was extensively investigated recently (8-10). Hydrogels are cross-linked networks of hydrophilic polymers capable of retaining large amounts of water yet remaining insoluble and maintaining their three-dimensional structure (11). However, the necessary criteria of hydrophilic polymers put limitations on their applications. And they have the disadvantage of burst release of drugs through breakdown of the matrices due to poor mechanical properties and high swelling ratio (12). Microcapsules have been developed as a convenient technology for drug delivery to protect encapsulated drugs from the external environment and control their release behaviors. They would be, in this regard, a promising candidate for the antimicrobial system. Yang H. prepared artemisia argyi oil-loaded antibacterial microcapsules by hydroxyapatite/poly (melamine formaldehyde) hybrid shells. They illustrated controlled release and long-term antimicrobial activity. However, the biocompatibility needs to be investigated furtherly (13). Tea tree oil-loaded antibacterial microcapsules were fabricated from sodium alginate/quaternary ammonium salt of chitosan by Mingjie Chen. High bacterial inhibition rate was demonstrated. And the surface roughness of microcapsules needed to be improved if applied for solid material release (14).

The Chinese nutgall (CN), which is a nature antibacterial extract without side effects, is getting more attention for biocidal functions (15). In Asian countries, it has been used for centuries in traditional medicines for treating inflammatory diseases. Water extract of $\mathrm{CN}$ is effective for inflamed tonsils, while direct application on skin cures swelling and inflammation. The constituents of $\mathrm{CN}$ are mainly hydrolysable tannins of tannic acid, gallic acid, ellagic acid and methyl gallate (16). Compared with synthetic antibacterial agents, $\mathrm{CN}$ is highly effective and nontoxic. To the best of our knowledge, although $\mathrm{CN}$ has been applied as antibacterial agents for long, most of them adopted CN extract directly. And CN still has great potential in long term-release or multi-functional antibiotics. Microcapsules are made up of the core and the shell. The shell has to stand various loads during fabrication and can degrade to release encapsulated materials. Sodium alginate (SA) is an anionic natural macromolecule, which is composed of poly- $\beta-1$, 4-d-mannuronic acid (M units) and $\alpha-1,4-1-$ glucuronic acid (G units) in varying proportions by 1-4 linkages. SA can be extracted from marine algae or produced by bacteria, and so it is abundant, renewable, non-toxic, water-soluble, biodegradable and biocompatible (17). As a cationic polymer derived chitin, chitosan is widely obtained. Besides, chitosan has great advantages of biocompatibility, biodegradability and hemostasis. It has been one of the most important antimicrobials against a broad spectrum of bacteria (18). It can also electrostatically interact with SA to form stable shells to protect materials encapsulated.

Thus, plant-derived $\mathrm{CN}$ was adopted as cores to fabricate biocompatible and long durability antimicrobial microcapsules by incorporating SA and chitosan as shells. All adopted materials are natural polymers. Cationic chitosan and anionic SA form stable shells to control the release of encapsulated CN. Chitosan can also provide biocidal functions. Besides, partial chitosan degradation or $\mathrm{CN}$ permeation through pores can release more extracts defending microbe. The microcapsules exhibit integrated properties of biocompatibility, long-term durability, high antibacterial efficacy and ease of storage. The incorporation of antibacterial microcapsules as a biocidal layer on wound dressings was demonstrated to achieve promising applications in wound dressing.

\section{Experimental}

\subsection{Experimental materials}

SA was obtained from Sinopharm Chemical Reagent Co., Ltd (Shanghai, China). Chitosan (deacetylation degree 92.36\%) was purchased in Jinke Biochemical Co., Ltd (Zhejiang, China). Calcium chloride $\left(\mathrm{CaCl}_{2}\right)$, glacial acetic acid $\left(\mathrm{CH}_{3} \mathrm{COOH}, 98 \%\right.$ in concentration), methyl alcohol $\left(\mathrm{CH}_{3} \mathrm{OH}\right)$, glutaraldehyde $(25 \%)$ and sodium hydroxide $(\mathrm{NaOH})$ were obtained from Lingfeng Chemical Reagent Co., Ltd (Shanghai, China), with analytical grade. CN extract was purchased from Huilin Bio-technology Co., Ltd (Shanxi, China).

\subsection{Preparation of the CN - SA/ chitosan microcapsules}

CN - SA/ chitosan microcapsules were fabricated by the orifice granulation method (19). Firstly, CN and SA chitosan solutions were prepared as follows. Solution A: $\mathrm{CN}$ extract was dissolved into $\mathrm{NaOH}$ solution, which was produced by dissolving $\mathrm{NaOH}$ in distilled water. Solution B: $\mathrm{NaOH}$ was dissolved in $\mathrm{CH}_{3} \mathrm{COOH}$ solution and 
the chitosan was added into the mixture. Secondly, the solution A was dropwise added into solution $\mathrm{B}$, and the system $(\mathrm{pH}=5.5)$ was stirred for $30 \mathrm{~min}$ at $45^{\circ} \mathrm{C}$. Thirdly, the glutaraldehyde was added to the reaction system to fix microcapsule shape. Finally, the suspension was dried in an electrothermal blowing dryer (Shanghai Yiheng Co., Ltd, China). The optimized parameters were attained by orthogonal experiment and shown in Table 1.

\subsection{CN encapsulation efficiency and stability}

The ultraviolet spectrum of $\mathrm{CN}$ in phosphate buffered saline (PBS, $\mathrm{pH}=7.7$ ) (20) was measured by UV- vis spectra photometer (TU-1901, Beijing Purkinje General Instrument Co., Ltd., Beijing, China). And the maximal absorption peak was at the wavelength of $256 \mathrm{~nm}$ (Figure S1). Accordingly, mass fraction - absorption intensity curve of CN was obtained (Figure S2). To calculate CN encapsulation efficiency, grinded microcapsules were dissolved in $\mathrm{CH}_{3} \mathrm{OH}$ and dispersed by ultra-sonication. After filtration, the solution absorption intensity at 256 m wavelength was recorded and $\mathrm{CN}$ encapsulation efficiency was calculated as the ratio of the weight of $\mathrm{CN}$ in microcapsules to theoretical loading $\mathrm{CN}$.

Besides, the storage stability of CN - SA/ chitosan microcapsules in $-20^{\circ} \mathrm{C}$ was also studied. At a predetermined time, $\mathrm{CN}$ in microcapsules was dissolved in PBS ( $\mathrm{pH}=7.7)$ and its absorption intensity was measured.

\subsection{Physical characterization of microcapsules}

The morphology of the $\mathrm{CN}$ loaded and unloaded microcapsules was observed by scanning electron microscopy (SEM; Jeol Ltd., Japan) at an accelerating voltage of $10 \mathrm{kV}$. Image processing software ImageJ

Table 1: The optimized parameters in preparation $\mathrm{CN}$ - SA/ chitosan microcapsules.

\begin{tabular}{lrrrr}
\hline $\begin{array}{l}\text { Microcapsule } \\
\text { coding }\end{array}$ & CC wt\% & SA wt\% & $\begin{array}{r}\text { Chitosan } \\
\text { wt\% }\end{array}$ & $\begin{array}{r}\text { SA wt\%: } \\
\text { CN wt\% }\end{array}$ \\
\hline $\mathrm{A}_{1}$ & 4 & 1.5 & 0.1 & $3.5: 1$ \\
$\mathrm{~B}_{1}$ & 3 & 1.5 & 0.3 & $3.5: 1$ \\
$\mathrm{C}_{1}$ & 4 & 2.0 & 0.1 & $3.5: 1$ \\
$\mathrm{D}_{1}$ & 5 & 1.0 & 0.5 & $3.5: 1$ \\
$\mathrm{~A}_{2}$ & 4 & 1.5 & 0.1 & $/$ \\
$\mathrm{B}_{2}$ & 3 & 1.5 & 0.3 & $/$ \\
$\mathrm{C}_{2}$ & 4 & 2.0 & 0.1 & $/$ \\
$\mathrm{D}_{2}$ & 5 & 1.0 & 0.5 & $/$ \\
\hline
\end{tabular}

was applied to measure the diameter of at least 50 microcapsules from at least 10 images. Fourier transform infrared (FTIR) spectra of different samples was recorded by absorption mode at $2 \mathrm{~cm}^{-1}$ intervals in the range of $4000-600 \mathrm{~cm}^{-1}$ wave numbers, using a FT-IR spectrophotometer (Avatar380, USA). Wide-angle X-ray diffraction (WAXD) of samples with and without CN was recorded by an X-ray diffractometer (Rigaku D/MAX 2550/ $\mathrm{PC}$, Japan) operating at $40 \mathrm{kV}$ and $200 \mathrm{~mA}$, along with $\mathrm{Cu}$ Ka radiation.

\subsection{In vitro release}

The release characteristics of $\mathrm{CN}$ from antimicrobial microcapsules were measured in PBS $(\mathrm{pH}=7.7)$ to simulate the $\mathrm{CN}$ release corresponding to the inflammation phase. Samples were placed in centrifuge tubes with the release medium at $37^{\circ} \mathrm{C}$. At predetermined time points, $1 \mathrm{~mL}$ solution of each sample was extracted to measure the absorption intensity at $256 \mathrm{~nm}$ wavelength. Meanwhile, an equal amount of fresh PBS was supplemented to each centrifuge tube. And the accumulative release rate (\%) was calculated as the ratio of released $\mathrm{CN}$ at each procedural time to the amount of loaded $\mathrm{CN}$.

\subsection{Antibacterial activity of microcapsules}

Agar diffusion plate test was applied to evaluate antibacterial activity of microcapsules qualitatively according to ISO 20645:2004 (20). Typical bacteria Grampositive Staphylococcus aureus (S. aureus) and Gramnegative Escherichia coli (E. coli) in wounds were selected as testing organisms (20) and their sensitivity was judged by the zone of inhibition. Microcapsule circle with the diameter of $20 \mathrm{~mm}$ was inoculated with approximately $1 \mathrm{~mL}$ of bacterial suspension $\left(\left(1 \times 10^{8} \mathrm{CFU} / \mathrm{mL}\right)\right.$ per tryptic soya agar plate. The plates were incubated at $37^{\circ} \mathrm{C}$ for $24 \mathrm{~h}$ and then measured by a Vernier caliper to determine the zone of inhibition, which was calculated as following formula (1),

$$
H=(D-d) / 2
$$

where $H$ is the zone of inhibition (mm), $D$ is the total diameter of the specimen and zone of inhibition $(\mathrm{mm})$ and $d$ is the diameter of the specimen ( $\mathrm{mm}$ ).

Oscillation method (GB/T 20944.3-2008, China Standards) was adopted to determine the antibacterial activity of microcapsules quantitatively. Inhibition rates (\%) were measured by: (control bacterial suspension $(\mathrm{CFU} / \mathrm{mL})$ - sample bacterial suspension $(\mathrm{CFU} / \mathrm{mL})) /$ control bacterial suspension $(\mathrm{CFU} / \mathrm{mL})$. 


\subsection{Application of microcapsules onto wound dressings and their antibacterial properties}

Cotton gauze is still a standard of care in the management of chronic wounds. It has reasonable gas permeability and effective exudates absorption (21). Here, we designed a composite wound dressing based on the cotton gauze and alginates. And antimicrobial microcapsules were applied on it (Figure 1). The outer cotton gauze layers helped exudates absorption, while the inner SA hydrogel provided a barrier for water vapor permeation. Antimicrobial microcapsules were fixed and incorporated into the SA hydrogel. Three layers were then laminated and antibacterial properties were evaluated according to agar diffusion plate test in ISO 20645:2004. For each sample, three specimens were tested.

\subsection{Statistical analysis}

Data reported was the means and standard deviations, and the error bars in figures corresponded to standard deviations. t-tests were used to determine the statistical difference. The data in the figures were marked by $\left({ }^{\star}\right)$ for $\mathrm{p}<0.05$.

\section{Results and discussion}

\section{1 $\mathrm{CN}$ encapsulation efficiency and stability}

The encapsulation efficiency of $\mathrm{CN}$ is an important index for CN loaded microcapsules and is shown in Figure 2a. SA and chitosan provide essential basis in the shell formation of microcapsules. Insufficient SA or chitosan may lead to low shell strength, or even shell breakage under foreigner loads. However, excessive SA can increase viscosity of the Solution $\mathrm{A}$ and then decrease $\mathrm{CN}$ encapsulation efficiency. $\mathrm{CaCl}_{2}$ works as a curing agent and its lack will

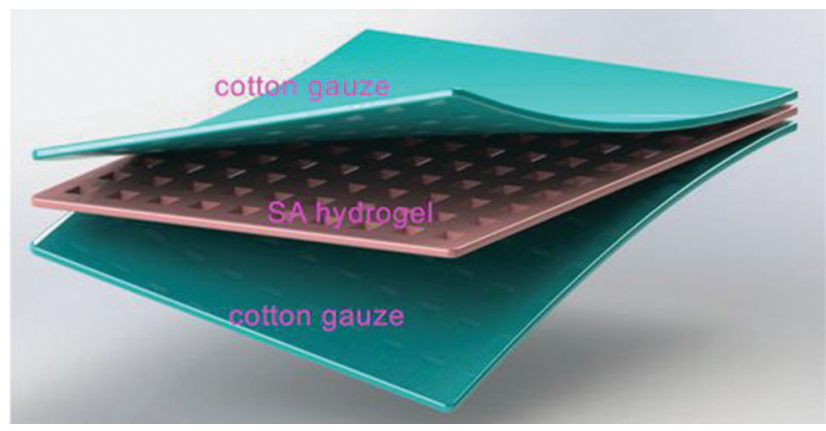

Figure 1: Composite wounding dressing. also cause shell softness, while rising amount can lead to thicker shell and less $\mathrm{CN}$ encapsulated. For the four optimized microcapsules by orthogonal method, they had encapsulation efficiencies of $80.16 \%, 74.21 \%, 70.79 \%$ and $70.11 \%$ for $\mathrm{A}_{1}, \mathrm{~B}_{1}, \mathrm{C}_{1}$ and $\mathrm{D}_{1}$, respectively.

Absorption intensity of $\mathrm{CN}$ after storage under $-20^{\circ} \mathrm{C}$ for different intervals is shown in Figure 2b. Compared with fluctuant intensity of $\mathrm{CN}$ extract, $\mathrm{CN}$ in microcapsules kept almost stable and unchanged. It indicated that microcapsules could protect $\mathrm{CN}$ from degradation and destruction due to electrostatic attraction between SA and chitosan.

\subsection{Physical characterization of microcapsules}

Microcapsules were successfully prepared by the orifice granulation method. After adding SA solution into chitosan solution, the interface became thicker as the aggregation caused by electrostatic attraction between the negative charges on SA and the positive charges of chitosan. And the above rigid shell was able to protect core materials. Figure 2c shows the images of microcapsules with good monodispersity, together with surface details. The surface of microcapsules with $\mathrm{CN}$ encapsulated is porous, which allows the release of $\mathrm{CN}$ to the surrounding medium. Cross-section image shows obvious layers of cores and shells. Microcapsules with core materials have more pores on the surface compared with microcapsules without core materials. Besides, the roughness of microcapsules increases with the decrease of encapsulation efficiency from $A_{1}$ to $D_{1}$ (22). Besides, mean size of $\mathrm{CN}$ - SA/ chitosan microcapsules are larger than microcapsules without $\mathrm{CN}$ included.

Encapsulation of $\mathrm{CN}$ is qualitatively confirmed by FTIR spectroscopy in Figure 3a. In the FTIR spectrum of the pure $\mathrm{CN}$, the peaks at $3457 \mathrm{~cm}^{-1}, 3153 \mathrm{~cm}^{-1}, 1722 \mathrm{~cm}^{-1}$ and $1617 \mathrm{~cm}^{-1}$ are attributed to stretching vibration of $\mathrm{O}-\mathrm{H}$, $\mathrm{C}-\mathrm{H}, \mathrm{C}=\mathrm{O}$ and $-\mathrm{C}=\mathrm{C}-$, respectively. Peaks at $1326 \mathrm{~cm}^{-1}, 1111$ $\mathrm{cm}^{-1}, 1039 \mathrm{~cm}^{-1}$ and $757 \mathrm{~cm}^{-1}$ are due to bending vibration of $-\mathrm{CH}_{3}, \mathrm{C}-\mathrm{O}-\mathrm{C}, \mathrm{C}-\mathrm{N}$ and $\bullet$, respectively. Compared with pure $\mathrm{CN}$, the FTIR spectrum of the $\mathrm{CN}$ loaded microcapsules has peaks at $1326 \mathrm{~cm}^{-1}, 1111 \mathrm{~cm}^{-1}, 1039 \mathrm{~cm}^{-1}$ and $757 \mathrm{~cm}^{-1}$, which derive from the $\mathrm{CN}$. Microcapsules without $\mathrm{CN}$ did not show any characteristic peaks of $\mathrm{CN}$. According to the results of FTIR spectra analysis, CN loaded microcapsules were prepared successfully.

Figure $3 \mathrm{~b}$ illustrates the changes in the intensity, height and weight of the reflection peak in $\mathrm{CN}$ extract and microcapsules. Sharp diffraction peak around $20^{\circ}$ indicates high crystallinity and larger crystal size inside 
(a)

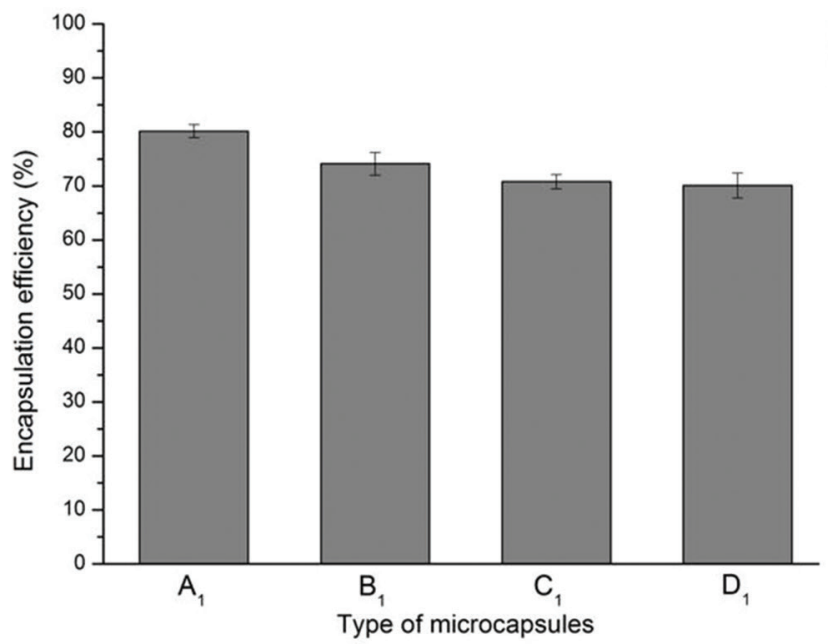

(c)
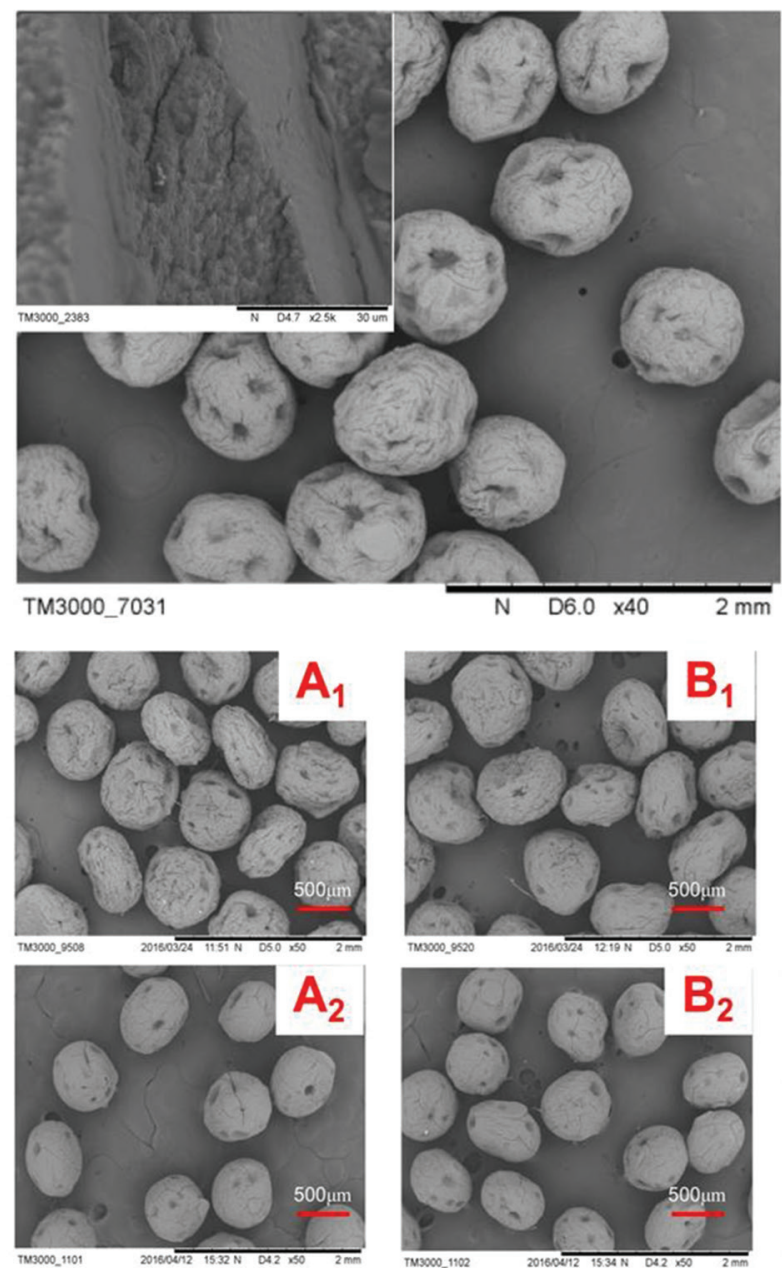

(b)
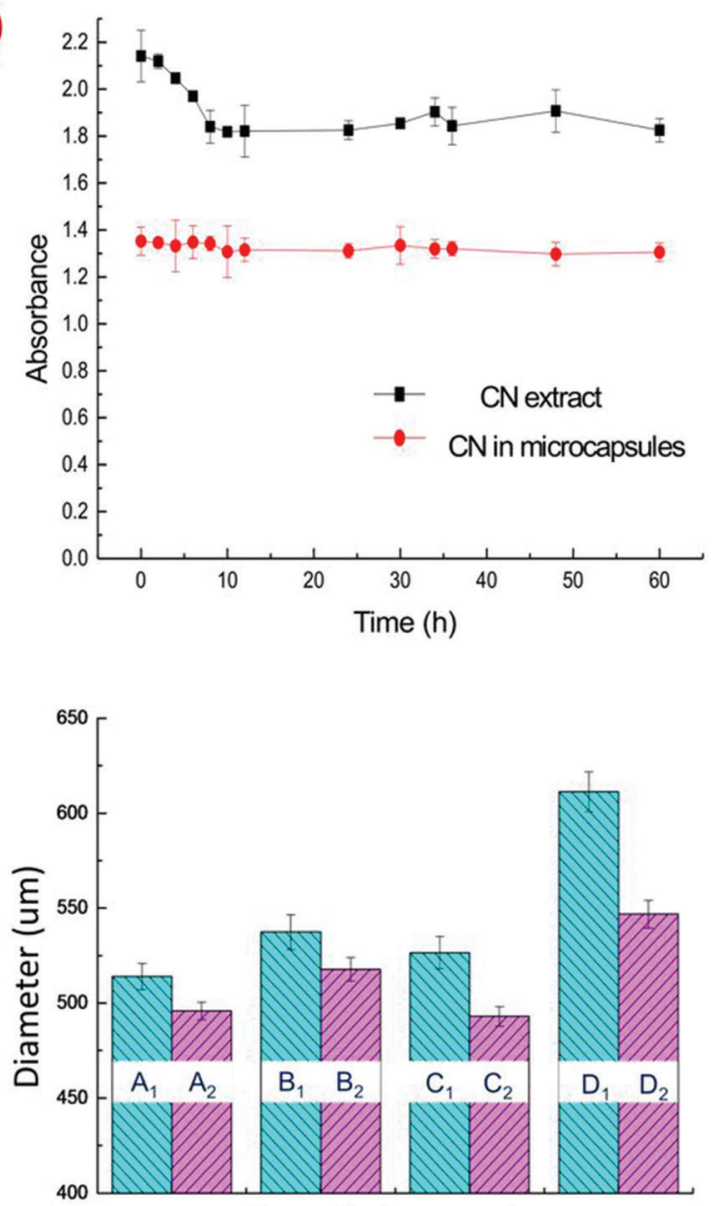

Type of microcapsules
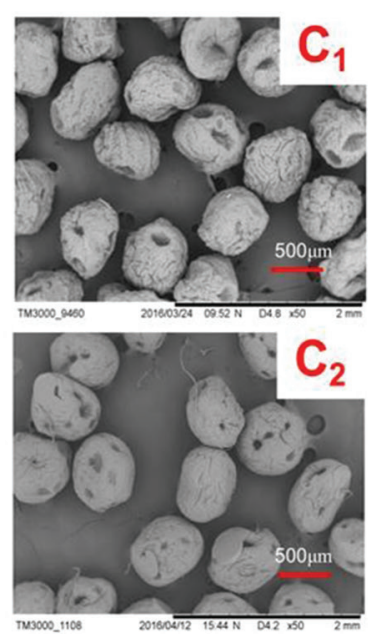
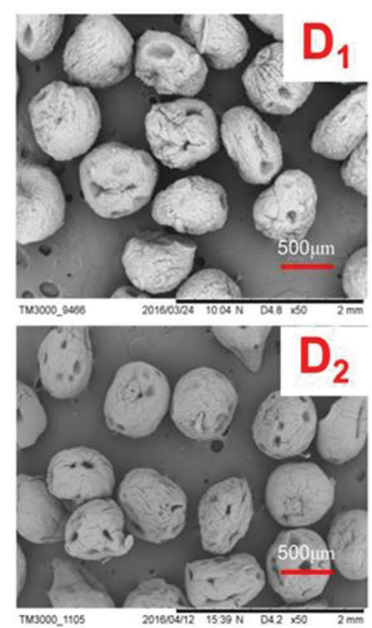

Figure 2: (a) Encapsulation efficiency of microcapsules; (b) Stability of microcapsules; (c) SEM images and average diameters of microcapsules.

CN. Peaks around $20^{\circ}$ in microcapsules confirmed the encapsulation of $\mathrm{CN}$, while shorter ones indicated $\mathrm{SA}$ and chitosan in shells affected the original structure of CN. On one hand, SA and chitosan interacted with each other by electrostatic attraction. On the other hand, carboxyl of SA and amidogen of chitosan can bond with phenolic hydroxyl groups in $\mathrm{CN}$, furtherly intervening with its crystal structures. 
(a)

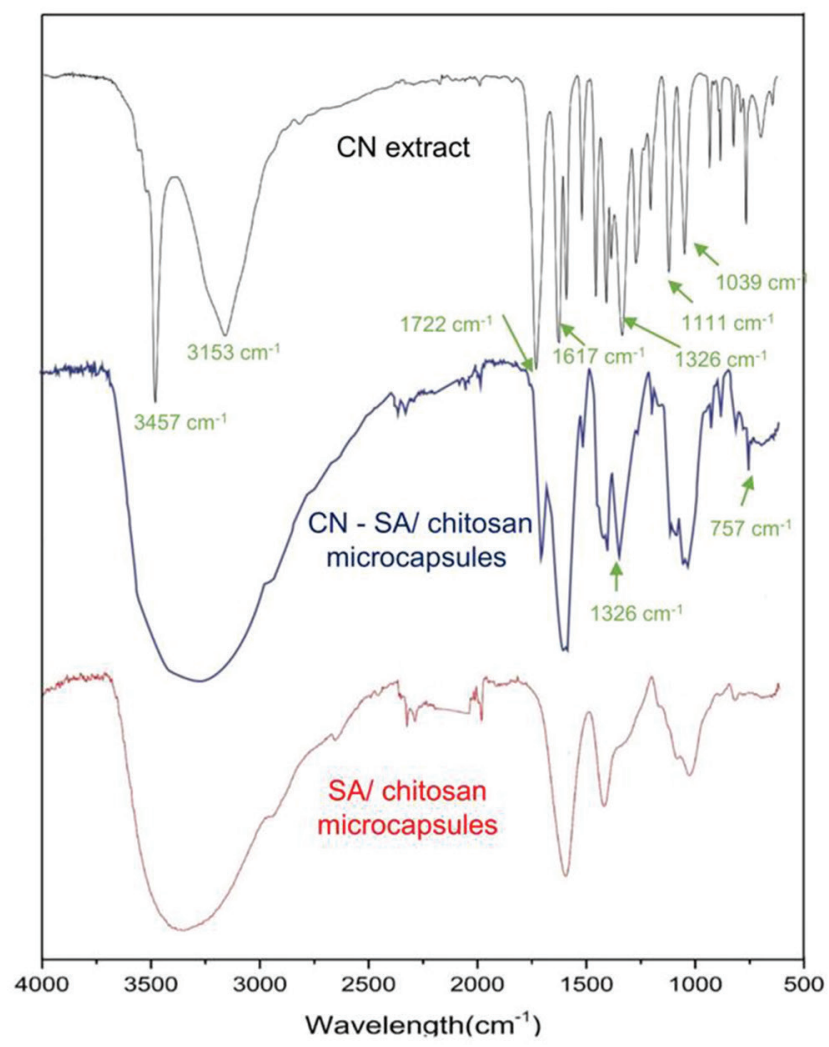

(b)

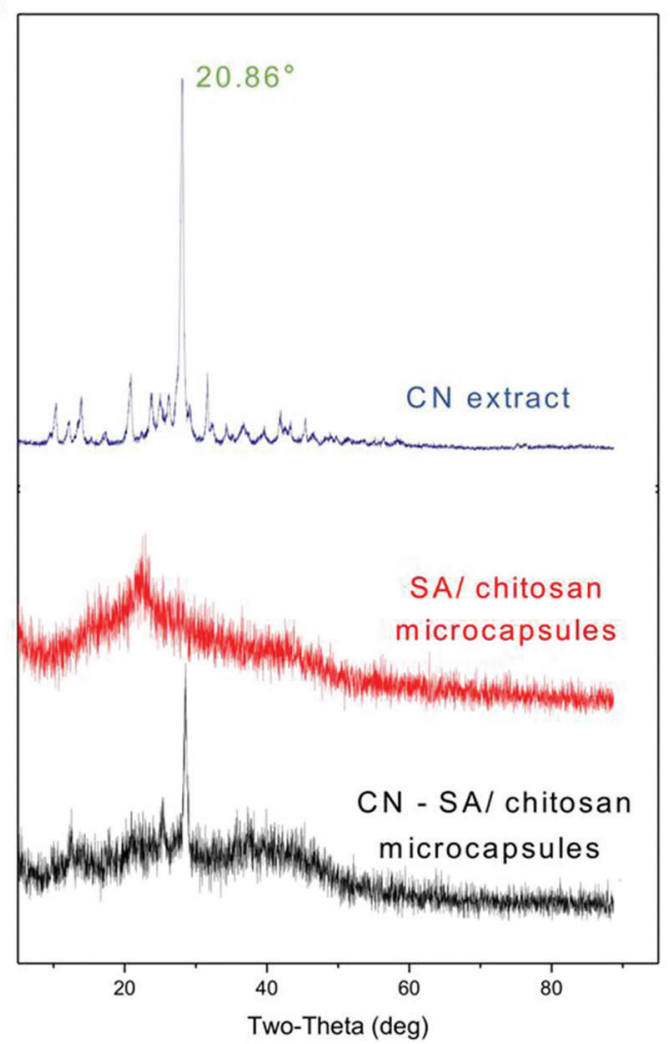

Figure 3: (a) FTIR spectroscopy of microcapsules; (b) XRD spectrum of microcapsules.

\subsection{In vitro release}

The release behaviors of $\mathrm{CN}$ from the microcapsules were investigated at $\mathrm{pH}=7.7$. Figure $4 \mathrm{a}$ gives a distinct picture of the release rate of $\mathrm{CN}$. It was seen that the release of $\mathrm{CN}$ demonstrated a continuous and comparatively even process over the study period. The initial "burst release" was around $20 \%$ for $B_{1}, C_{1}$ and $D_{1}$, and $35 \%$ for $A_{1}$, which was very weak, during the first $12 \mathrm{~h}$. It was primarily attributed to the protection of $\mathrm{CN}$ by shells. $\mathrm{CN}$ sustaining release reached the release proportions around $80 \%$ after $60 \mathrm{~h}$. It is the basis of long-term durability of microcapsule antibacterial activity.

Acute wound healing process can be divided into four periods, among which inflammation phase is more likely to cause microorganism growth (23). In this period, the skin $\mathrm{pH}$ increases from 5-6 to 7 or more (24). In acid medium, the COO- groups in alginates transform into $\mathrm{COOH}$ groups. On the one hand, the hydrogen-bonding interaction among $\mathrm{COOH}$ groups is strengthened and the additional physical crosslinking is generated; on the other hand, the electrostatic repulsion among COO- group is restricted, and so the alginate shell tends to shrink. $\mathrm{CN}$ release from the core decreases (25). But partial chitosan degrades to defend microbes at the first stage. However, in inflammation stage, the skin $\mathrm{pH}$ increases in wounds and the ratio of COO- groups in polymer network increase. Consequently, the hydrogen-bonding interaction is broken and the electrostatic repulsion among negatively charged polymer chains increases, and so the polymer network tends to swell. Degraded chitosan also leads to more pores for $\mathrm{CN}$ release to kill the bacteria.

\subsection{Microcapsule antibacterial activity}

Antibacterial activity of microcapsules with CN loaded and unloaded against $S$. aureus and E. coli was investigated. The results (Figure $4 \mathrm{~b}$ ) revealed that CN-loaded microcapsules had strong antibacterial effects, with $S$. aureus inhibition zones of $7.67 \mathrm{~mm}$ and $E$. coli inhibition zones of $5.27 \mathrm{~mm}$ after incubation for $24 \mathrm{~h}$. Microcapsules without $\mathrm{CN}$ encapsulation also illustrated antimicrobial property, with $S$. aureus inhibition zones of $3.25 \mathrm{~mm}$ and $E$. coli inhibition zones of $1.33 \mathrm{~mm}$, which were the contributions of chitosan. In addition, it was observed that the antibacterial effect against $E$. coli was slightly weak compared to $S$. aureus. Quantitative evaluations (Figure 4c) also illustrated higher inhibition rates 
against $S$. aureus. Besides, antibacterial performance of microcapsules increased with the $\mathrm{CN}$ encapsulation rates, while microcapsules without $\mathrm{CN}$ performed similar inhibition rates. It confirmed the biocidal functions of CN. The inhibition rate could research $98.99 \%$ against S. aureus after $12 \mathrm{~h}$ and $100 \%$ after $24 \mathrm{~h}$ (Table S1) for microcapsule $\left(\mathrm{A}_{1}\right)$ loading concentration of $4.5 \mathrm{mg} / \mathrm{mL}$. It remained $99.61 \%$ against $E$. coli after $6 \mathrm{~h}$ and kept $100 \%$ after $12 \mathrm{~h}$ (Table S2) for $5.0 \mathrm{mg} / \mathrm{mL}$, demonstrating a longterm antibacterial performance.

So, at the early stage of wound healing, chitosan in microcapsule shells plays the role to defend bacteria and help hemostatic. At inflammation stage, alginate networks swell with the increase of skin $\mathrm{pH}$ and partial chitosan degrades with pores left. Thus, more $\mathrm{CN}$ in the core release to defend microbe.

\subsection{Wound dressing antibacterial properties}

Antimicrobial performance of composite wound dressings was investigated and shown in Figure 5. CN loaded dressings have $S$. aureus inhibition zones of $4.82 \mathrm{~mm}$ and $E$. coli inhibition zones of $4.11 \mathrm{~mm}$, while dressings without $\mathrm{CN}$ showed $S$. aureus inhibition zones (a)

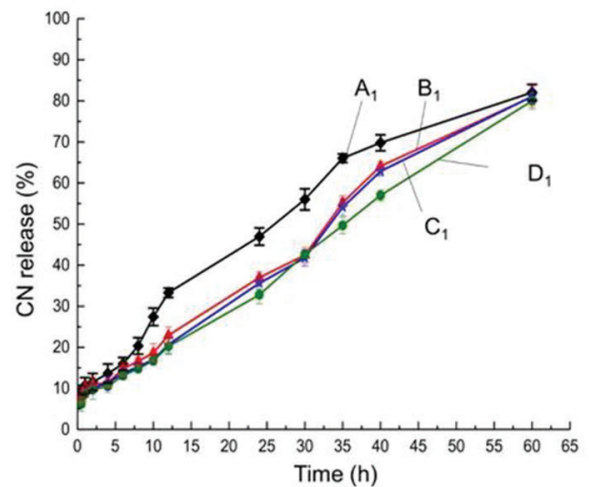

(b)

\begin{tabular}{|c|c|c|c|}
\hline $\begin{array}{l}\text { Microcapsule } \\
\text { type }\end{array}$ & Bacteria & $H(\mathrm{~mm})$ & $\begin{array}{l}\text { Bacteria growth } \\
\text { description }\end{array}$ \\
\hline $\begin{array}{l}\text { CN-SA/chitosan } \\
\text { microcapsules }\end{array}$ & S. aureus & $7.67 \pm 0.56$ & Inhibited totally \\
\hline $\begin{array}{l}\text { SA/chitosan } \\
\text { microcapsules }\end{array}$ & S. aureus & $3.25 \pm 0.88$ & Some colony left \\
\hline $\begin{array}{l}\text { CN-SA/chitosan } \\
\text { microcapsules }\end{array}$ & E. coli & $5.27 \pm 0.47$ & Inhibited totally \\
\hline $\begin{array}{l}\text { SA/chitosan } \\
\text { microcapsules }\end{array}$ & E. coli & $1.33 \pm 0.96$ & Inhibited almost \\
\hline
\end{tabular}

(c)
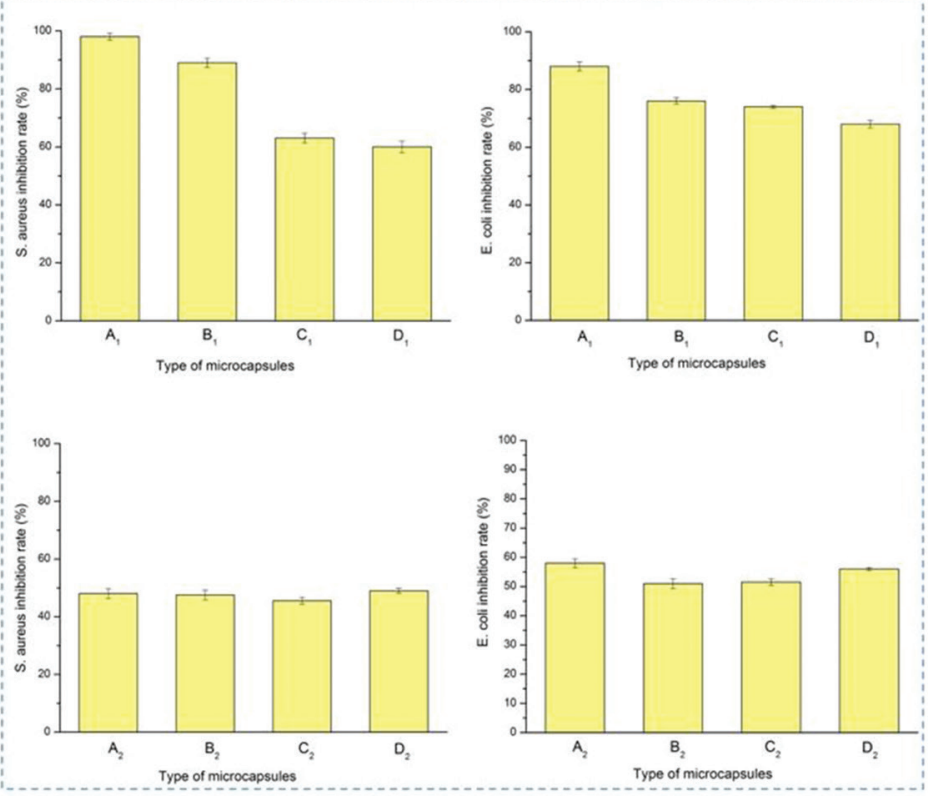

Figure 4: (a) CN release of microcapsules along with the time; (b) Microcapsule qualitative antibacterial activity; (c) Microcapsule quantitative antibacterial activity.
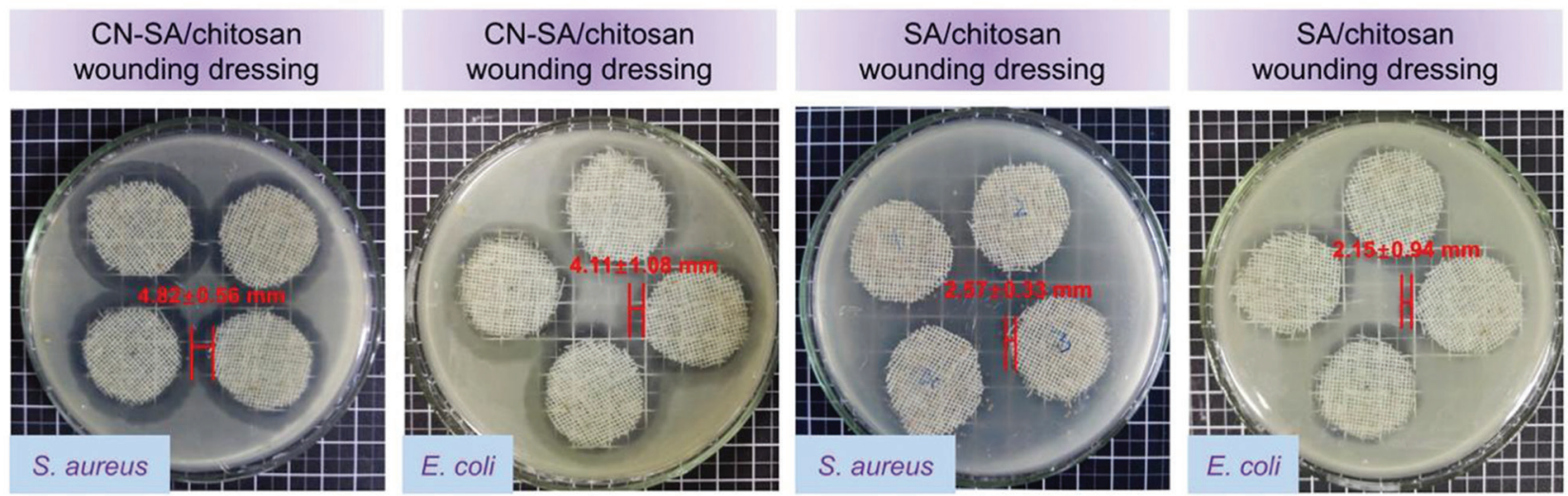

Figure 5: Wound dressing antibacterial properties. 
of $2.57 \mathrm{~mm}$ and $E$. coli inhibition zones of $2.15 \mathrm{~mm}$. Because of indirect $\mathrm{CN}$ contact with bacteria, wound dressings have inferior antimicrobials compared with microcapsules. According to ISO 20645:2004, they still showed good antibacterial effect. Moreover, cotton gauze on the outer layer works as wicking of excessive exudation. Alginates in the middle can fix antibacterial microcapsules, as well as provide a barrier for water vapor permeation by forming hydrogels through ionic exchange. The whole system works cooperatively to help wound healing.

\section{Conclusions}

In conclusion, we have demonstrated the antibacterial performance of plant-derived $\mathrm{CN}$ and its incorporation in microcapsules. The obtained microcapsules had rough surfaces and high stability under $-20^{\circ} \mathrm{C}$. Besides, prominent antimicrobial effects of $\mathrm{CN}$ as well as its sustained release profile was observed. Chitosan in microcapsule shells can defend bacteria and help hemostatic at the early stage of wound healing. At the inflammation stage, alginate networks swell and degraded chitosan lead to more $\mathrm{CN}$ release to combat infections. Thus, the developed microcapsules with natural formed a biocompatible and long durability antibacterial system, which was promising in wound dressing applications.

Acknowledgement: The project is support by the Fundamental Research Funds for the Central Universities (grant No. 2232017A-05, 2232018G-01), Science and Technology Support Program of Shanghai (grant No. 16441903803), the Chinese Universities Scientific Fund (grant No. CUSF-DH-D-2017012), National Health and Family Planning Commission Fund (No. 2017ZX01001-S22) and 111 project (grant No. B07024).

Supplementary material: Figure S1 (a) The ultraviolet spectrum of $\mathrm{CN}$ in PBS ( $\mathrm{pH}=7.7)$; (b) $\mathrm{CN}$ concentration - absorption curve. Table S1 Inhibition rates of microcapsules against $S$. aureus. Table S2 Inhibition rates of microcapsules against E. coli.

Conflicts of interests: The authors declare no conflict of interest.

Author contributions: M. Z., F. W., J. G. and L. W. conceived and designed the experiments. W. X. and M. $Z$. performed the experiments including fabrication and analysis. W. X. wrote the paper.

\section{References}

1. Velnar T., Bailey T., Smrkolj V., The wound healing process: an overview of the cellular and molecular mechanisms. J Int Med Res, 2009, 37(5), 1528-1542.

2. Unnithan A. R., Barakat N.A.M., Pichiah P.B.T., Gnanasekaran G., Nirmala R., Cha Y.S., et al., Wound-dressing materials with antibacterial activity from electrospun polyurethane-dextran nanofiber mats containing ciprofloxacin $\mathrm{HCl}$. Carbohyd Polym, 2012, 90(4), 1786.

3. Liu J., Liu C., Liu Y., Chen M., Yang H., Yang Z., Study on the grafting of chitosan-gelatin microcapsules onto cotton fabrics and its antibacterial effect. Colloid Surface B, 2013, 109(9), 103-108.

4. Si Y., Zhang Z., Wu W., Fu Q., Huang K., Nitin N., et al., Daylightdriven rechargeable antibacterial and antiviral nanofibrous membranes for bioprotective applications. Sci Adv, 2018, 4(3), eaar5931.

5. Palaniappan K., Holley R.A., Use of natural antimicrobials to increase antibiotic susceptibility of drug resistant bacteria. International Journal of Food Microbiology, 2010, 140(2), 164-168.

6. Cisowska A., Wojnicz D., Hendrich A. B., Anthocyanins as antimicrobial agents of natural plant origin. Nat Prod Commun, 2011, 6(1), 149-156.

7. Dorman H., Deans S.G., Antimicrobial agents from plants: antibacterial activity of plant volatile oils. J Appl Microbiol, 2000, 88(2), 308-316.

8. Masood F., Polymeric nanoparticles for targeted drug delivery system for cancer therapy. Mat Sci Eng C, 2016, 60, 569.

9. Bharti C., Nagaich U., Pal A.K., Gulati N., Mesoporous silica nanoparticles in target drug delivery system: A review. Int J Pharm Investig, 2015, 5(3), 124-133.

10. Xue X., Zhao Y., Dai L., Zhang X., Hao X., Zhang C., et al., Spatiotemporal drug release visualized through a drug delivery system with tunable aggregation-induced emission. Adv Mat, 2014, 26(5), 712-717.

11. Vermonden T., Censi R., Hennink W. E., Hydrogels for Protein Delivery. Chem Rev, 2012, 112(5), 2853.

12. Wang Q., Zhang J.P., Wang A.Q., Preparation and characterization of a novel $\mathrm{pH}$-sensitive chitosan--poly (acrylic acid)/attapulgite/ sodium alginate composite hydrogel bead for controlled release of diclofenac sodium. Carbohyd Polym, 2009, 78(4), 731-737.

13. Hu Y., Yang Y., Ning Y., Wang C., Tong Z., Facile preparation of artemisia argyi oil-loaded antibacterial microcapsules by hydroxyapatite-stabilized Pickering emulsion templating. Colloid Surface B, 2013, 112, 96-102.

14. Chen M., Hu Y., Zhou J., Xie Y., Wu H., Yuan T., et al., Facile fabrication of tea tree oil-loaded antibacterial microcapsules by complex coacervation of sodium alginate/quaternary ammonium salt of chitosan. RSC Adv, 2016, 6(16), 1303213039.

15. Wang C.G., Chinese pulsatilla extracts eliminate resistance of Escherichia coli to streptomycin. Afr J Microbiol Res, 2011, 5(21), 81-4.

16. Pithayanukul P., Nithitanakool S., Bavovada R., Hepatoprotective potential of extracts from seeds of Areca catechu and nutgalls of Quercus infectoria. Molecules, 2009, 14(12), 4987-5000.

17. Wang W., Wang A., Synthesis and swelling properties of $\mathrm{pH}$-sensitive semi-IPN superabsorbent hydrogels based on 
sodium alginate--poly(sodium acrylate) and polyvinylpyrrolidone. Carbohyd Polym, 2010, 80(4), 1028-1036.

18. Gupta B., Arora A., Saxena S., Alam M.S., Preparation of chitosan\&ndash;polyethylene glycol coated cotton membranes for wound dressings: preparation and characterization. Polym Advan Technol, 2010, 20(1), 58-65.

19. Zheng L.J., Luo Y.P., Wang Y.J., Xing-Guo H.U., Zhi-Qing W.U., Bao F.K., et al., Advances in the study of the antibacterial and anti-inflammatory action of Galla chinensis. Journal of Pathogen Biology, 2011, 6(11), 868-1105.

20. Chen X., Hou D., Wang L., Zhang Q., Zou J., Sun G., Antibacterial SurgicalSilkSutures Using a High-Performance Slow-Release Carrier Coating System. Acs Appl Mater Inter, 2015, 7(40), 22394-22403.

21. Edwards J.V., Yager D. R., Cohen I.K., Diegelmann R.F., Montante S., Bertoniere N., et al., Modified cotton gauze dressings that selectively absorb neutrophil elastase activity in solution. Wound Repair Regen 2001, 9(1), 50.

22. Zhang M., Rao B., Gao J., Wang F., Wang L., Wu J., et al., Preparation and structure research of gallnut antibacterial microcapsules. Industrial Textiles, 2016, 34(7), 28-34.

23. Witte M.B., Barbul, A., General principles of wound healing. Surg Clin N Am, 1997, 77(3), 509.

24. Schneider L.A., Korber A., Grabbe S., Dissemond J., Influence of $\mathrm{pH}$ on wound-healing: a new perspective for wound-therapy? Arch Dermatol Res, 2007, 298(9), 413-420.

25. Seeli D.S., Dhivya S., Selvamurugan N., Prabaharan M., Guar gum succinate-sodium alginate beads as a $\mathrm{pH}$-sensitive carrier for colon-specific drug delivery. Int J Biol Macromol, 2016, 91, 45-50. 